\title{
Publisher's Note: Investigating Anisotropic Quantum Hall States with Bimetric Geometry [Phys. Rev. Lett. 119, 146602 (2017)]
}

\author{
Andrey Gromov, Scott D. Geraedts, and Barry Bradlyn \\ Q (Received 6 February 2018; published 23 February 2018)
}

DOI: 10.1103/PhysRevLett.120.089902

This Letter was published online on 5 October 2017 with an error in the Acknowledgments on page 5. On page 5, left-hand column, the third sentence in the last paragraph should read as "A. G. was supported by the Leo Kadanoff fellowship and NSF Grant No. DMR-1206648." The Acknowledgments have been corrected as of 13 February 2018. The Acknowledgments are incorrect in the printed version of the journal. 